\title{
Decay of the Turbulent Cascade of Capillary Waves on the Charged Surface of Liquid Hydrogen
}

\author{
G. V. Kolmakov, A. A. Levchenko, M. Yu. Brazhnikov, \\ L. P. Mezhov-Deglin, A. N. Silchenko*, and P. V. E. McClintock* \\ Institute of Solid State Physics RAS, 142432, Chernogolovka, Russia \\ ${ }^{*}$ Department of Physics, Lancaster University, Lancaster, LA1 $4 Y B$, UK
}

We study the free decay of capillary turbulence on the charged surface of liquid hydrogen. We find that the decay begins from the high frequency spectral domain of the surface oscillations and is of a quasi-adiabatic character. The characteristic relaxation time of the whole turbulent cascade is close to the viscous damping time for capillary waves of frequency equal to the driving frequency.

PACS: 47.27.-i, 47.25.+i

We report and discuss the main results of an investigation into the decay of turbulence in a system of capillary waves on the charged surface of liquid hydrogen following removal of an external driving force. This work represents an extension of our recent studies of steady-state capillary turbulence ${ }^{1,2}$.

It is known that the turbulent behavior of waves on the surface of a liquid can be well-described within the framework of weak turbulence theory ${ }^{3}$. In this approach the statistical properties of an ensemble of surface waves are characterized by "occupation numbers" $n_{\omega}$ for wave modes of frequency $\omega$, obeying the kinetic equation

$$
\frac{\partial n_{\omega}}{\partial t}=S t\left[n_{\omega}\right]-2 \gamma_{\omega} n_{\omega}+F_{\text {drive }}(t) .
$$

Here $S t\left[n_{\omega}\right]$ is the collision integral, $\gamma_{\omega}=2 \nu(\rho / \sigma)^{1 / 3} \omega^{4 / 3}$ is the coefficient of viscous damping of the surface waves, $F_{\text {drive }}(t)$ is the external low frequency driving force, $\nu$ is the coefficient of viscosity, $\sigma$ is the surface tension, and $\rho$ is the density of the liquid. For capillary waves, where the dispersion relation $\omega=(\sigma / \rho)^{1 / 2} k^{3 / 2}$ is of the decay type, it is the interaction of three surface 


\section{G. V. Kolmakov et al.}

waves that makes the main contribution to the collision integral (i.e. the decay of one wave into two waves, and the opposite process of confluence of two waves into one wave).

In the inertial frequency range (i.e. much higher then the driving frequency, but lower than the frequency scale where the dissipation plays the dominant role in the wave dynamics) the turbulent behavior of capillary waves is determined mainly by their nonlinear interaction. For wideband pumping of the liquid surface the Kolmogorov turbulence spectrum $n_{\omega} \sim Q^{1 / 2} \omega^{-15 / 6}$ becomes established, where the energy flux $Q$ is directed towards the high frequency domain.

In our earlier experiments ${ }^{1,2}$ on the charged surface of liquid hydrogen, we observed and studied capillary turbulence over a rather wide range of frequencies (from $100 \mathrm{~Hz}$ to $10 \mathrm{kHz}$ ). The remarkable properties of liquid hydrogen - its small density, low viscosity, high nonlinearity of capillary waves, and the possibility of driving the electrically charged surface of the liquid directly - allowed us to establish for the first time how the scaling exponent of the turbulence distribution depends on the spectral characteristics of the driving force, and to observe the high frequency cut-off of the power spectrum of capillary turbulence caused by the change in the energy transfer mechanism from nonlinear wave transformation to viscous damping ${ }^{2}$.

In what follows we describe a new investigation of the decay of capillary turbulence on the charged surface of liquid hydrogen, seeking to establish what happens following a step-like removal of the driving force.

The experimental arrangements were similar to those used in our earlier studies of steady state turbulence ${ }^{4}$. The measurements were made using an optical cell inside a helium cryostat. Hydrogen was condensed into a cup formed by a bottom capacitor plate and a guard ring $60 \mathrm{~mm}$ in diameter and $6 \mathrm{~mm}$ high. The layer of liquid was $6 \mathrm{~mm}$ thick. The top capacitor plate (a collector $60 \mathrm{~mm}$ in diameter) was located at a distance of $4 \mathrm{~mm}$ above the surface of the liquid. A two-dimensional positive charge layer was created just below the surface of the liquid with the aid of a radioactive plate placed at the bottom of the cup. The temperature of the liquid was $15.5 \mathrm{~K}$. The waves on the charged surface were excited by a periodic driving voltage applied between the guard ring and the upper electrode. They were detected from the variation of the total power $P(t)$ of a laser beam reflected from the oscillating surface, which was measured with a photodetector, sampled with an analogue-to-digital converter, and stored in a computer. Given the size of the light spot, the correlation function $I_{\omega}=\left\langle\left|\eta_{\omega}\right|^{2}\right\rangle$ of the surface elevation $\eta(\mathbf{r}, t)$ in frequency representation is directly proportional to the squared modulus of the Fourier transform of the detected signal, $I_{\omega}=\operatorname{const} P_{\omega}^{2}$ at frequencies above $50 \mathrm{~Hz}$. 
Decay of the Turbulent Cascade of Capillary Waves
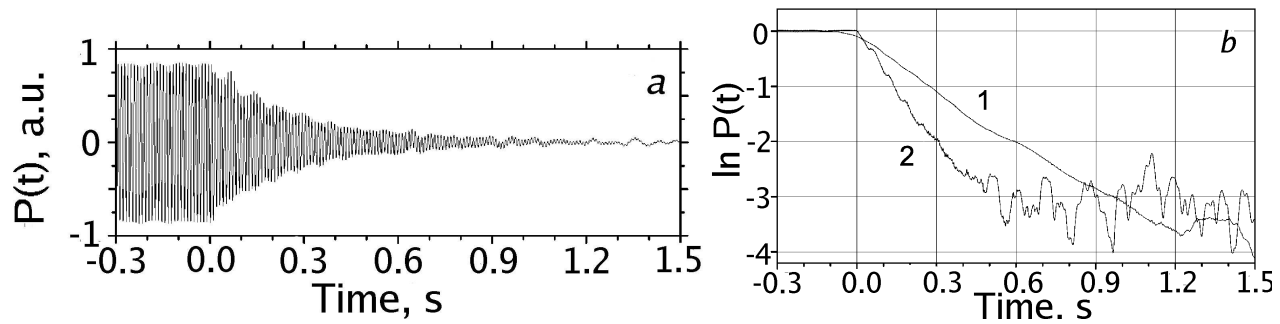

Fig. 1. (a) Relaxation of oscillations of the charged liquid hydrogen surface after removal of external driving at frequency $\omega_{p} / 2 \pi=97 \mathrm{~Hz}$ at time $t=0$. (b) Dependence on time of the amplitude of the recorded signal $P(t)$ : curve 1 for a driving frequency $\omega_{p} / 2 \pi=97 \mathrm{~Hz}$; and curve 2 for $\omega_{p} / 2 \pi=173 \mathrm{~Hz}$.

In systems of finite size, the power spectrum of capillary turbulence is discrete; but, at frequencies much higher than the lowest resonant frequency ( $\sim 3 \mathrm{~Hz}$ in our experiments), the intrinsic spectrum of resonant frequencies becomes quasi-continuous due to viscous broadening of the resonances and/or nonlinear broadening for pumping rates exceeding a critical value. Under these conditions the distribution of turbulent oscillations of the surface of liquid in a cell of finite dimensions is close to that expected for the unrestricted surface (see theory ${ }^{5,6}$ and observations ${ }^{1,2,7,8}$ ). To establish the steady turbulent state at the surface of the liquid, a $95 \mathrm{~Hz}$ ac driving voltage was applied for $\sim 10 \mathrm{~s}$. After it was switched off, we observed relaxation oscillations of the surface. The instantaneous power spectrum $P_{\omega}^{2}$ of the nonstationary surface oscillations was calculated by application of a short-time Fourier transform ${ }^{9}$ to the measured signal $P(t)$.

Fig. 1(a) shows a fragment of the experimental signal $P(t)$ from a photodetector recorded when driving at a frequency $\omega_{p} / 2 \pi=97 \mathrm{~Hz}$. The driving force was removed at time $t=0$. For $t>0$ we observe the relaxation of surface oscillations (decreasing signal amplitude). Fig. 1(b) shows the dependence of the signal amplitude on time, after driving at frequencies $\omega_{p} / 2 \pi=97 \mathrm{~Hz}$ (see Fig. 1(a)) and $\omega_{p} / 2 \pi=173 \mathrm{~Hz}$. The data presented in Fig. 1(b) were obtained by averaging the modulus of the signal $P(t)$ over. It is found that the decreasing signal amplitude following removal of the driving force could be well-described by the exponential function $P(t) \sim \exp (-t / \tau)$, where the effective relaxation time $\tau=(0.3 \pm 0.03) \mathrm{s}$ for driving frequency $\omega_{p} / 2 \pi=97 \mathrm{~Hz}$ and $\tau=(0.15 \pm 0.02) \mathrm{s}$ for frequency $\omega_{p} / 2 \pi=173 \mathrm{~Hz}$. The dependence of the effective relaxation time $\tau$ on frequency $\omega_{p} / 2 \pi$ is shown by filled circles in Fig. 2. The straight line shows the dependence of the viscous damping time for capillary waves $\tau_{\omega}=\gamma_{\omega}^{-1}$ at frequency $\omega=\omega_{p}$, calculated from the known parameters ${ }^{10}$ of liquid hydrogen at $15.5 \mathrm{~K}$. It is 


\section{G. V. Kolmakov et al.}

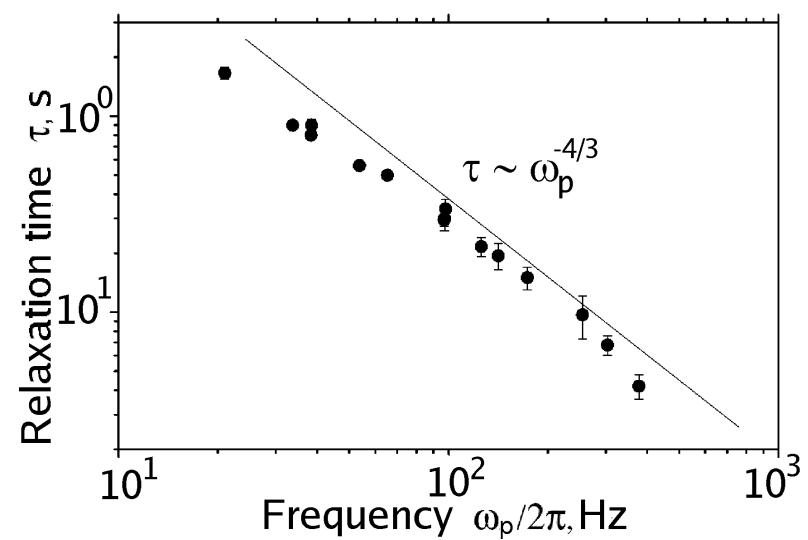

Fig. 2. Dependence of the effective relaxation time $\tau$ of the surface oscillations on the driving frequency $\omega_{p}$ (filled circles); and viscous damping time for capillary wave $\gamma_{\omega}^{-1}$ with frequency $\omega=\omega_{p}$, calculated from known parameters of liquid hydrogen (line).

clearly evident from Fig. 2 that the measured relaxation time $\tau$ is close to the viscous damping time of the waves.

Fig. 3 shows the instantaneous spectra $P_{\omega}$ calculated for times (a) $\mathrm{t}=0.34 \mathrm{~s}$ and $(\mathrm{b}) \mathrm{t}=1.07 \mathrm{~s}$ after removal of the driving force. At times close to $t=0$ the calculated spectrum of surface oscillations is close to the steady-state distribution of capillary waves observed in earlier experiments using narrow-band driving ${ }^{2}$; the initial (steady-state) boundary frequency of the inertial interval was $\omega_{b} / 2 \pi \approx 5 \mathrm{kHz}$. The main spectral peak in Fig. $3(\mathrm{a})$ is at the driving frequency of $97 \mathrm{~Hz}$, and the peaks at harmonics of the driving frequency form a cascade with a power dependence $P_{\omega}^{2} \sim \omega^{-3.5}$ of the peak amplitudes, in accordance both with our previous observations ${ }^{2}$ and with theory ${ }^{3}$. The arrow marks the high frequency boundary of the inertial range. It is evident from Fig. 3(a) that the boundary frequency of the inertial interval has moved down from its initial value $\omega_{b} / 2 \pi \approx 5 \mathrm{kHz}$ at $t=0$ to $\omega_{b} / 2 \pi=2 \mathrm{kHz}$ at $t=0.35 \mathrm{~s}$. Fig. 3(b) shows the final stage in the decay of turbulence at $t=1.07 \mathrm{~s}$, when only a few harmonics are excited.

It is remarkable that the low-frequency part of the spectrum of surface oscillations can still be well-described by the same power law $P_{\omega}^{2} \sim \omega^{-3.5}$ as is valid for the steady-state turbulent distribution during the relatively long time interval $t<0.6 \mathrm{~s}$ after removal of the driving force. The decay of the turbulence starts from the high frequency domain of the spectrum and is accompanied by movement of the high frequency boundary $\omega_{b}$ of the inertial interval towards the low frequency domain. It can clearly be seen in Fig. 3 that the amplitude of the surface oscillations at the driving frequency 
Decay of the Turbulent Cascade of Capillary Waves
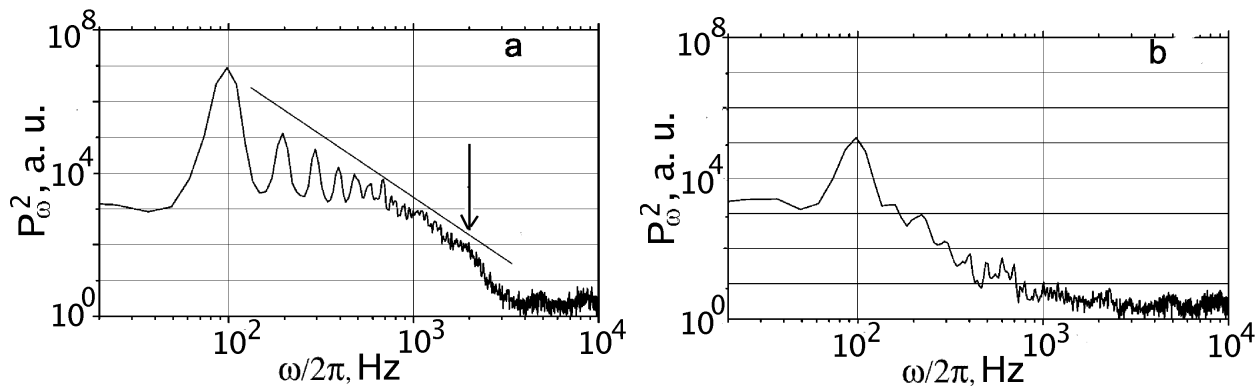

Fig. 3. Instantaneous spectra of surface oscillations calculated for different times following removal of the driving force: (a) at $t=0.35 \mathrm{~s}$; (b) at $t=$ $1.07 \mathrm{~s}$. The straight line corresponds to the power dependence $P_{\omega}^{2} \sim \omega^{-7 / 2}$. The arrow in (a) marks the position of the high frequency boundary of the inertial range.

exceeds noticeably the amplitudes of waves at the harmonics at all times during the decay. These observations differ significantly from what could be expected from the self-similar theory of nonstationary turbulent processes ${ }^{3}$, which was developed for cases where the dissipation is strictly equal to zero. Note that we do not observe any transition processes (for example, front propagation from low to high frequencies) after removal of the driving force. This means that the presence of finite viscous damping of the waves causes a qualitative change in how the system of nonlinear surface waves relaxes. We may infer that the shape of the low frequency (power-like) part of the spectrum is stabilized by a fast redistribution of energy between waves within the inertial range of frequencies. This is why the observed spectrum remains close to quasiequilibrium over relatively long times following the start of the decay.

By numerical integration of the kinetic equation (1) we have shown ${ }^{11}$ that, at large times, the decrease in amplitude of low frequency waves is mainly on account of viscous damping; nonlinear interaction with high frequency oscillations is negligibly small due to the smallness of the amplitudes of the high frequency waves. Waves of frequency much higher than the driving frequency are excited by interaction with low frequency waves. This means that under the conditions of real experiments (i.e. for finite width of the inertial range, limited by viscosity at frequencies of $5-10 \mathrm{kHz}$ ) the nonlocal interaction between capillary waves is comparable with this local interaction of waves. This accounts convincingly for the observed peculiarities in the decay of capillary turbulence on the surface of liquid hydrogen. In particular it explains why the dependence on the driving frequency of the effective relaxation time of surface oscillations is close to the dependence 


\section{G. V. Kolmakov et al.}

typical of linear waves.

In conclusion, after removal of a low frequency driving force, we observe a quasi-adiabatic decay of the turbulent cascade of capillary waves on the surface of liquid hydrogen. The decay starts from high frequency domain of the spectrum, and the effective relaxation time of the cascade is close to the viscous damping time for waves of frequency equal to the driving frequency. The energy-containing range stays in the low frequency domain throughout the decay. This indicates that viscous losses at all frequencies are of central importance for the correct interpretation of relaxation processes in a system of nonlinearly interacting capillary waves.

The authors are very grateful to V.E. Zakharov, E.A. Kuznetsov and M.T. Levinsen for many useful discussions, and to V.N. Khlopinskii for assistance in preparation of the experiments. The investigations are supported in part by INTAS (grant 2001-0618), by RFBR (grant 03-02-16865), by the Ministry of Industry, Science and Technology of RF (program "Quantum phenomena at low and ultralow temperatures"), by the Leverhulme Trust (UK), and by the Engineering and Physical Sciences Research Council (UK). G.V.K. acknowledges also support from the Science Support Foundation (Russia).

\section{REFERENCES}

1. M. Brazhnikov, A. Levchenko, G. Kolmakov, and L. Mezhov-Deglin, JETP Lett. 73, 398 (2001).

2. M. Brazhnikov, A. Levchenko, and G. Kolmakov, JETP 95, 447 (2002).

3. V. Zakharov, V. L'vov, and G. Falkovich, Kolmogorov Spectra of Turbulence, Vol. 1 (Springer, Berlin, 1992).

4. M. Yu. Brazhnikov, A. A. Levchenko, and L. P. Mezhov- Deglin, Instr. Exp. Techn. 45, 758 (2002).

5. A. N. Pushkarev and V. E. Zakharov, Phys. Rev. Lett. 76, 3320 (1996).

6. A. I. Dyachenko, A. O. Korotkevich, and V. E. Zakharov, JETP Lett. 77, 477 (2003).

7. W. Wright, R. Budakian, and S. Putterman, Phys. Rev. Lett. 76, 4528 (1996).

8. E. Henry, P. Alstrom, and M. T. Levinsen, Europhys. Lett. 52, 27 (2000).

9. S. Mallat, A Wavelet Tour of Signal Processing (Academic Press, New York, 1997); R. Gribonval and E. Bacry, IEEE Trans. Signal Processing 51, 101 (2003).

10. B. I. Verkin, ed., Properties of Condensed Phases of Hydrogen and Oxygen (Kiev, Naukova Dumka, 1984).

11. G. V. Kolmakov, A. A. Levchenko, M. Yu. Brazhnikov, L. P. Mezhov-Deglin, A. N. Silchenko, and P. V. E. McClintock, "Quasi-adiabatic decay of capillary turbulence on the charged surface of liquid hydrogen", Phys. Rev. Lett. 93 (7), 074501 (2004), in press. 